Bull. Austral. Math. Soc.

$11 \mathrm{E} 92,15 \mathrm{~A} 63,05 \mathrm{~B} 20$

VOL. 40 (1989) [303-321]

\title{
ON BILINEAR FORMS REPRESENTED BY TREES
}

\author{
Walter D. NeumanN
}

To Father, with love.

\begin{abstract}
The adjacency matrix of a weighted graph deternines an integral bilinear form. The trees with uninodular adjacency matrices are described with special emplasis on the definite and semidefinite cases, since they arise as configuration graphs of good divisors in compact complex surfaces.
\end{abstract}

A weighted forest is a finite forest (finite simple graph with no cycles) with an integer weight $e_{v}$ assigned to each vertex $v$; its connected components are weighted trees. Associated with a weighted forest $\Gamma$ is a symmetric bilinear form $A_{\Gamma}$ on the free Z-inodule freely generated by the vertices of $\mathbf{l}$ :

$$
A_{\Gamma}(v, w)= \begin{cases}e_{v} & \text { if } v=w \\ 1 & \text { if } v \text { is connected to } w \text { by an edge } \\ 0 & \text { otherwise }\end{cases}
$$

We write det $\Gamma:=\operatorname{det} A_{\Gamma}$. Our aim is to describe the weighted trees $\Gamma$ with $\operatorname{det} \Gamma=$ \pm 1 , which we call unimodular trees. A famous example of such a tree is

$$
E_{8}=0-0 \text { (all vertices weighted }-2 \text { ). }
$$

Although the unimodular case is our primary interest, some of our results apply with no restriction on $\mid$ det $\Gamma \mid$.

There are certain elementary operations that one can do to a weighted forest without changing |alet $\Gamma \mid$, and we first describe the classification of weighted forests modulo the equivalence relation generated by these operations. Denote by $\left(p_{+} \Gamma, p_{-} \Gamma\right):=$ $\left(p_{+}\left(A_{\Gamma}\right), p_{-}\left(A_{\Gamma}\right)\right)$ the index of the bilinear form $A_{\Gamma}$ (number of positive, respectively negative, entries in a diagonalisation of the form over $\mathbf{P})$. Then in each equivalence class of weighted forests there is a "normal form" characterised by the fact that it has the smallest $p_{+}$amongst all forests in the equivalence class and is, in addition, the

Received 13 April 1989

Research partially supported by the NSF and the Max-Planck-Institut für Malhematik in Bonn.

Copyright Clearance Centre, Inc. Serial-fee code: 0004-9729/89 \$A2.00+0.00. 
smallest forest with this property. We compute the minimal $p_{+}$for any equivalence class of unimodular forests (in [3] the ones with $p_{+}=0$ were characterised). This allows a fairly practical description of the unimodular trees and forests having any prescribed $p_{+}$. We carry this out for $p_{+}=0,1$.

Our title is "bilinear forms represented by trees," but it will be clear by now that we are emplasising the trees more than the forms. Indefinite unimodular symmetric forms $A$ over $Z$ are easily classified (they are diagonalisable if odd-that is $A(x, x)$ is odd for some $x$-and equivalent to an orthogonal sum of copies of $\left(\begin{array}{ll}0 & 1 \\ 1 & 0\end{array}\right)$ and $\pm A_{E_{B}}$ otherwise, see for example [11]), so only in the definile case is the form per se interesting. In the final section we discuss briefly the classification of negative definite forms represented by trees, but we cannot say inuch. The following conjecture from [8] indicates our degree of ignorance.

Conjec'rune. If the form $A_{\Gamma}$ represented by a tree $\Gamma$ is equivalent over $Z$ to the form diag $(-1,-1, \ldots,-1)$, then some vertex of $I^{\prime}$ has weight -1 .

If $-\Gamma$ denotes the reversal of sign of all weights of $\Gamma$, then the form $A_{-\Gamma}$ is equivalent to $-A_{\Gamma}$ (use a basis transformation that just reverses the signs of basis elements at alternate vertices of $\Gamma$ ). Thus 1 could be replaced by -1 in the above conjecture and positive definite forests become negative definite on reversing the signs of all weights. The negative definite bias of this paper follows that of the weighted graphs that arise in algebraic geometry: negative definite graphs arise as resolution diagrams for complex surface singularities; graphs with $p_{+}=1$ arise as configuration diagrams of divisors in compact complex surfaces. Such a weighted graph is a unimodular tree if and only if the 3-dimensional link of the corresponding gepmetric object is a homology sphere. 'There has been recent interest in this geometric situation, for example $[\mathbf{1 0}, \mathbf{6}$, 8].

The material on classification (Sections 1 and 3 ) is in the literature (in part just implicitly- $[5,12,3])$, but the existing proofs have topological ingredients. For the most part we only sketch how one may replace these proufs by purely combinatorial prools, but we have tried to make the paper otherwise self-contained and readable without topological background.

\section{OPERATIONS ON TREES AND FORESTS.}

First some terminology. If $\Gamma$ is a weighted tree, the valency of a vertex of $\Gamma$ is the nuuber of edges at that vertex; a node is a vertex of valency $\geqslant 3$. If the nodes are removed from $\mathbf{\Gamma}$, then $\Gamma$ falls into comnected components which are called the chains of 1 ; they consist of strings of vertices of valency 1 or 2 connected by edges. An isolated vertex (valency 0 ) is also a chain. 
The following operations replace a tree $\Gamma$ by a smaller tree or forest without changing |det $A_{1} \mid$. The first operation, blowing down, eliminates a $( \pm 1)$-weighted vertex of valency $\leqslant 2$, reducing the number of vertices by 1 ; the second and third operations, 0 -absorption and splitting, eliminate a 0 -weighted vertex of valency 2 or 1 , reducing the number of vertices by 2 . The pictures are meant to suggest unspecified numbers of additional edges, maybe zero, meeting the indicated $a$-weighted and $b$-weighted vertices.

$\varepsilon$-Blowdown $(\varepsilon= \pm 1)$

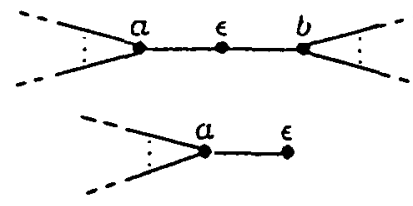

$\epsilon$

0-Absorption

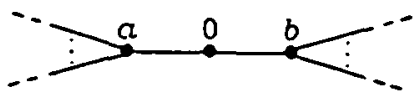

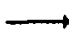
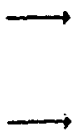

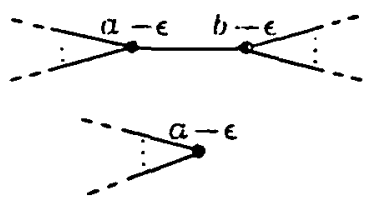

$\not$

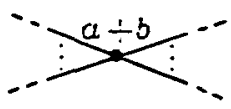

Splitting (this replaces a tree by a forest of $k \geqslant 0$ trees)
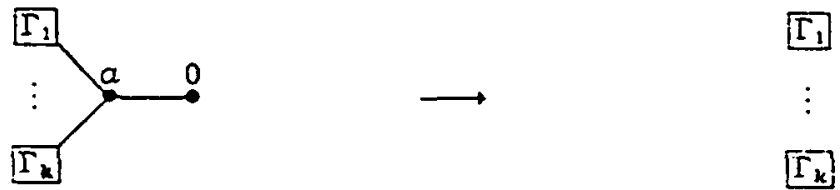

Any of these operations can be performed on a forest by acting on a component tree of the forest. The inverse operations are called $\varepsilon$-blowup, 0 -extrusion, and connecting, respectively.

Proposition 1.1. The above operations have the following effect on $A_{\Gamma}$

$$
\left.\begin{array}{cl}
\begin{array}{c}
(-1)-b l o w d o w n: \\
(+1)-b l o w d o w n:
\end{array} & \operatorname{det} \Gamma \rightarrow-\operatorname{det} \Gamma,\left(p_{+} \Gamma, p_{-} \Gamma\right) \rightarrow\left(p_{+} \Gamma, p_{-} \Gamma-1\right) \\
\text { 0-absorption }: \\
\text { splitting: }
\end{array}\right\} \quad \begin{aligned}
& \operatorname{det} \Gamma \rightarrow-\operatorname{det} \Gamma,\left(p_{+} \Gamma, p_{-} \Gamma\right) \rightarrow\left(p_{+} \Gamma-1, p_{-} \Gamma\right) \\
&
\end{aligned}
$$

Proof: It is easily verified that the effect on $A_{\Gamma}$ of the inverse operations is to add orthugonally the matrix $(1),(-1)$ or $\left(\begin{array}{ll}b & 1 \\ 1 & 0\end{array}\right)$ respectively, and then perform a simple basis change. 
We shall call two trees or forests equivalent if they are related by a sequence of the above operations and their inverses. We call them strictly equivalent if they are related by $(-1)$-blowup and (-1)-blowdown operations only. Thus equivalent forests have the same $\mid$ det $\Gamma \mid$ and strictly equivalent ones have the same $p_{+} \Gamma$ also.

We shall say $\Gamma$ satisfies the negative chains condition if (NCC) any vertex on a chain (that is of valency $\leqslant 2$ ) has negative weight. and we say $\mathrm{l}$ ' is in normal form if (MIN) Any vertex on a chain has weight $\leqslant-1$, that is the negative chains condition hulds and no blowdown is possible.

ThEOREM 1.2. Among the $\Gamma$ in a given equivalence class there is a minimal value for $p_{+} \Gamma$. The $\Gamma$ in the equivalence class which attain this ninimal value of $p_{+}$satisfy the negative chains condition and are strictly equivalent to each other. Anong these $\Gamma$ with minimal $p_{+}$there is a unique smallest, characterised by the fact that it is in normal form.

Corollary 1.3. If $\Gamma$ has minimal $p_{+}$in its equivalence class, it is obtained from its normal form by repeated (-1)-blowups.

PROOF: If $\Gamma$ satisfies negative chains but is not in normal form then it admits a (-1)-blowdown, and by iterating we must reach the normal form.

Proof of Theorem 1.2: Suppose $\Gamma$ does not have negative chains. Then there is a weight $b \geqslant 0$ on some chain, so by performing $b(-1)$-blowups next to this weight we can create a weight 0 and by then performing a 0 -alssorption or splitling we get an equivalent forest with smaller $p_{+}$. Thus a forest with minimal $p_{+}$in its equivalence class has negative chains.

The theorem now follows from the topological results of [5] as follows. In [5] it is shown that if $M(\Gamma)$ is the oriented 3-manifuld constructed by "plumbing according to I'" then

(1) equivalent weighled forests give the same $M(\Gamma)$;

(2) there is a unique $\Gamma_{0}$ in normal form with $M\left(\Gamma_{0}\right)=M(\Gamma)$;

(3) $\Gamma_{0}$ can be obtained from $\Gamma$ by repeated operations of the following types: $(-1)$-blowup, $(-1)$-blowdown, $(+1)$-blowdown, 0-absorption and splitting.

Note tliat, by Proposition 1.1, no operation in (3) increases $p_{+}$, so $p_{+} \Gamma_{0} \leqslant p_{+} \Gamma$. Moreover, if $p_{+} \Gamma_{0}=p_{+} \Gamma$ then no $(+1)$-bluwdown, 0 -absorption or splitting was involved, so $I_{u}^{\prime}$ is strictly equivalent to $\Gamma$. This proves the theorem.

One can give a combinatorial proof of the theorem by replacing $M(\Gamma)$ by a combinatorial object which satisfies (1), (2) and (3) above. We will not give this proof 
in delail-it is a bit tedious-but we describe a suitable combinatorial object $W(\Gamma)$, called a $W$-graph (these are basicly the graphs Waldhausen originally used to classify "graph-manifolds" in [13]). A $W$-graph is a forest $\Delta$ with no vertex of valency 0 or 2 , and with the following extra structure

(a) $\Delta$ has an integer wejght at each node;

(b) associated with each direcled edge $e$ of $\Delta$ is a coprime integer pair $\left(p_{e}, q_{e}\right)$ such that if $e^{\prime}$ is the same edge oppositely directed then $p_{e}=p_{e^{\prime}}$ and $q_{e} q_{e^{\prime}} \equiv 1\left(\bmod p_{e}\right)$;

(c) the weights $\left(p_{e}, q_{e}\right)$ satisfy $0 \leqslant q_{e}<p_{e}$,

(d) if $e$ is not an edge connecting two nodes then $p_{e}>1$.

The $W$-graph associated with a normal form forest $\Gamma$ can be constructed as follows. The underlying graph of $W(\Gamma)$ is obtained by replacing each maximal chain of $\Gamma$ by a single edge. Weights at nodes are unclianged. Given a maximal chain

$$
\underset{a}{-b_{1}-b_{2}}+\cdots-b_{k}
$$

in $\Gamma$, the corresponding edge of $W(\Gamma)$ directed from left to right is given the weight $(p, q)$ with $p / q=\left[b_{1}, \ldots, b_{k}\right]$ in lowest terms, where

$$
\left[b_{1}, \ldots, b_{k}\right]=b_{1}-\frac{1}{b_{2}-\frac{1}{\ddots \cdot-\frac{1}{b_{k}}}},[]=\frac{1}{0}
$$

Even if $\Gamma$ is not in normal form, the above procedure might give a $W$-graph, in which case it is the currect $W$-graph, but in general the resulting graph will violate one or both of conditions (c) and (d) and the correct $W$-graph is obtained by a suitable "reduction" procedure. We shall not need this reduction procedure and its delails are not hard to work out, so we just sketch them. The main ingredients are: for an edge starling from a node $v$ whose $q_{e}$ does not salisly $(\mathrm{c}),\left\lfloor q_{e} p_{e}\right\rfloor$ is subtracted from the weight at $v$ and then $q_{e}$ is reduced $\bmod p_{e}$; edges with $p_{e}=0$ or 1 are collapsed in a similar way to the blowdown, absorption and splitting operations for weighted trees; if the edge $e$ has $p_{e}=0$ one must first add the number $\left[0, b_{1}, \ldots, b_{k-1}\right]$, which is an integer, to the weight of an adjacent node. (It is helpful-but not essential-to work out how to deal with vertices of valency 2 in an "unreduced" $W$-graph. The original weighted tree can then be thought of as an unreduced $W$-graph with weight $(1,0)$ on cvery edge.)

For unimodular forests there is a much more convenient classifying object than the $W^{\prime}$-graph. We will describe this in Section 3. 


\section{Determinants and Continued fraction.}

A rooted tree is a tree with a particular root vertex picked out. Suppose $\Gamma$ is a rooted weighted tree whose root $v$ has valency $d$. If we delete $v$ and its adjacent edges, we oblain a forest $\bar{\Gamma}$ which is the disjuint union of trees $\Gamma_{1}, \ldots, \Gamma_{d}$, each of which is rooted by taking the vertex of $\Gamma_{i}$ adjacent to $v$ as the root. We can repeat: let $\overline{\bar{\Gamma}}=\bigcup_{i=1}^{d} \bar{\Gamma}_{i}$, et cetera. Define the continued fraction of $\Gamma$ to be

$$
\text { cf } \Gamma=\frac{\operatorname{det} \Gamma}{\operatorname{det} \bar{\Gamma}} \in Q \cup\{\infty\}
$$

where we put $x / 0=\infty$ for any $x \in Q$.

Proposition 2.1. If the weight at the root vertex $v$ is $b$ then

$$
\operatorname{det} \Gamma=b \operatorname{det} \bar{\Gamma}-\sum_{i=1}^{d}\left(\operatorname{det} \bar{\Gamma}_{i} \prod_{j \neq i} \operatorname{det} \Gamma_{j}\right),
$$

so

$$
\text { cf } \mathrm{r}=b-\sum_{i=1}^{d} \frac{1}{\mathrm{cf} \Gamma_{i}}
$$

Proof: Equation (2) is an elementary computation: expand the determinant of $A_{\Gamma}$ according to the row corresponding to $v$. Equation (3) follows by dividing by $\operatorname{det} \bar{\Gamma}=\prod_{i} \operatorname{det} \Gamma_{\boldsymbol{i}}$. This equation plus induction implies

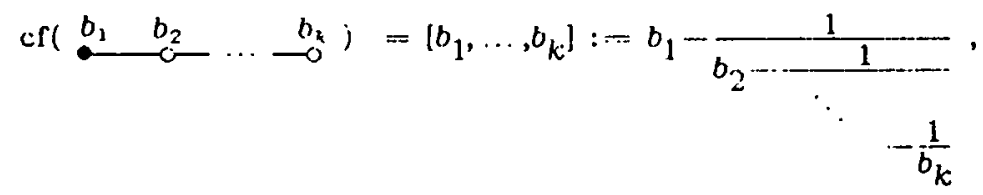

which justifies the name "continued fraction."

Conollary 2.2. If $\operatorname{gcd}(\operatorname{det} \Gamma, \operatorname{det} \bar{\Gamma})=1$ then $\operatorname{gcd}\left(\operatorname{det} \Gamma_{i}, \operatorname{det} \bar{\Gamma}_{i}\right)=1$ for all $i$ and $\operatorname{gcd}\left(\operatorname{det} \Gamma_{i}, \operatorname{det} \Gamma_{j}\right)=1$ for all $i \neq j$.

Proof: This is immediate from equation (2), since $\operatorname{det} \bar{\Gamma}=\prod_{i} \operatorname{det} \Gamma_{i}$.

For each vertex $u$ of $\Gamma$, let $\Gamma_{u}$ be the full subtree on vertices $w$ such that the direct path from $w$ to the root $v$ passes through $u$. We lake $u$ as the root of $\Gamma_{u}$. In particular, $\Gamma_{v}=\Gamma$. These subtrees are the ones obtained by taking components of $\Gamma$, $\bar{\Gamma}, \overline{\bar{\Gamma}}$, et cetera, so applying Curollary 2.2 repeatedly gives: 
Corollary 2.3. If $\operatorname{gcd}(\operatorname{det} \Gamma, \operatorname{det} \bar{\Gamma})=1$ then $\operatorname{gcd}\left(\operatorname{det} \Gamma_{u}, \operatorname{det} \bar{\Gamma}_{u}\right)=1$ for all vertices $u$ of $\Gamma$, so det $\Gamma_{u}$ and det $\bar{\Gamma}_{u}$ are numerator and denominator of ef $\Gamma_{u}$ up to sign.

Note that a unimodular tree certainly satisfies $\operatorname{gcd}(\operatorname{det} \Gamma$, det $\bar{\Gamma})=1$. The standard computation of the index of a form in terms of sign changes of its major subdeterminants shows:

CoROLlary 2.4. If $\Gamma$ is unimodular then $p_{+} \Gamma$ and $p_{-} \Gamma$ are respectively the number of positive and negative of $\Gamma_{u}$ as $u$ ranges over the vertices of $\Gamma$ ( $\infty$ is counted as both positive and negative for this).

If no ef $\Gamma_{u}=\infty$ occurs then this Corollary can also be seen by noting that if one diagonalises $A_{\Gamma}$, starting from the leaves and working back to the root, the diagonal entries are the of $\mathrm{r}_{u}$.

\section{SPLICE DIAGRAMS}

A splice diagram is a forest weighted in the following manner

- each node has a weight +1 or -1 (which we often just write as "+" or "-");

- at each node there is an integer weight associaled with each edge incident at the node;

- at each node these "edge weights" are pairwise coprine.

The following is an example of a splice diagram.

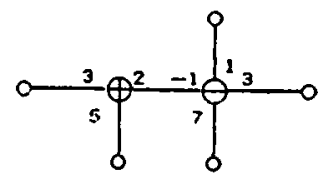

Two splice diagrams are called equivalent if one results from the other by a sequence of operations or inverses of operations of the following types

- simultaneously change the signs of an even number of weights (sign weight and edge weights) at any given node;

- if an edge from a node to a leaf (a vertex of valency 1 ) has edge weight 1 , then delete it and its leaf;

- if an edge from a node to a leaf has edge weight 0 , then delete the node, all edges incident on the node, and the lear;

- replace any vertex of valency 2 and its two adjacent edges by a single edge.

- discard any component with 2 or less vertices; 
- given an edge connecting two nodes of a splice diagram with weights as follows ( $\varepsilon$ and $\varepsilon^{\prime}$ are the vertex weights);

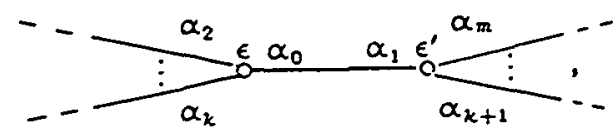

sucl that $\varepsilon \varepsilon^{\prime} \alpha_{0} \alpha_{1}-\alpha_{2} \cdots \alpha_{m}=0$, replace the edge by a single node

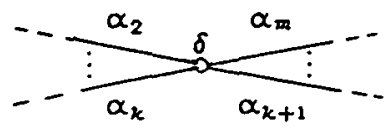

with $\delta=\varepsilon \varepsilon^{\prime} \operatorname{sign}\left(\alpha_{0} \alpha_{1}\right)$.

'The above number $\varepsilon \varepsilon^{\prime} \alpha_{0} \alpha_{1}-\alpha_{2} \cdots \alpha_{m}$ is called the edge determinant for the given edge.

We shall say a splice diagram is reduced if none of the above operations except the first (allowable sign changes) is applicable and is in normal form if it is reduced and, moreover, all edge weights are non-negative and the vertex weight adjacent to any 0 edge weight is +1 . It is easy to see that any splice diagram is equivalent to a unique normal form splice diagram. For example, the splice diagram (1) above has normal form

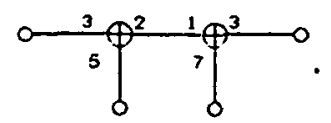

Suppose now that $\Gamma$ is a unimodular tree. We form a connected splice diagram $\Delta=\Delta(\Gamma)$ from $\Gamma$ as follows. The underlying tree of $\Delta$ is obtained by replacing each maximal chain in $\Gamma$ by a single edge. Given a node $v$ of $\Gamma$ of valency $d$, weight the $d$ edges of $\Delta$ around $v$ by the determinants det $\left(-\Gamma_{1}\right), \ldots, \operatorname{det}\left(-\Gamma_{d}\right)$, where the $\Gamma_{i}$ are the components of the result of deleting $v$ and its incident edges from $\Gamma$. Finally all nodes are given the weight det $(-\Gamma)$, which is \pm 1 by assumption. For a unimodular forest $\Gamma$ we form $\Delta(\Gamma)$ by applying this procedure component by component to $\Gamma$.

THeORem 3.1. [3, Chapter V]. The above construction $\Gamma \mapsto \Delta(\Gamma)$ defines a bijection between unimodular forests up to equivalence and splice diagrams up to equivalence. If $\Gamma$ is in normal form then the resulting splice diagram $\Delta$ is reduced.

Proof: This is proved in [3], but a combinatorial proof is not hard, so we sketch the main ingredients. Firstly, $\Delta\left(\Gamma^{\prime}\right)$ is indeed a splice diagram because the edge weights at a node are pairwise coprime by Corollary 2.2. It is an easy verification to see that the alluwable operatious to change $l^{\top}$ within its equivalence class translate to allowable 
operations for $\Delta(\Gamma)$, so that $\Gamma \mapsto \Delta(\Gamma)$ gives a well-defined map of sets of equivalence classes.

Now suppose that $\Gamma$ is in normal form, or even just "reduced" in the sense that it adınils no blowdown, 0 -absorption, or splitting operation. Then a computation which we onit shows that $\Delta(\Gamma)$ is reduced. The main ingredient in this computation is that the edge determinant of an internal edge or the edge weight of an extremal edge of $\Delta(\Gamma)$ is, up to sign, the numerator of the continued fraction of the corresponding chain in $\Gamma$ (this is the $p_{e}$-weight on the edge of the corresponding $W$-graph). Its absolute value therefore exceeds the length of the corresponding chain in $\Gamma$.

It remains to describe how to reconstruct a unimodular weighted forest from a splice diagram. We describe this in detail since we need it later. It is enough to construct a unimodular weighted tree $\Gamma(\Delta)$ from a given connected normal form splice diagram $\Delta$. We shall do this by "splicing together" pieces corresponding to simpler sub-diagrams of $\Delta$, starting with splice diagrams with a single node. We cannot assume that these sub-diagrams are in normal form in that they may have extremal edges with edge weight 0 or 1 . If

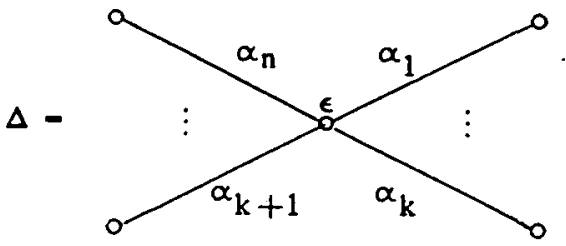

where $\varepsilon= \pm 1$ is the vertex weight and $\alpha_{i} \geqslant 0$ for each $i$, then

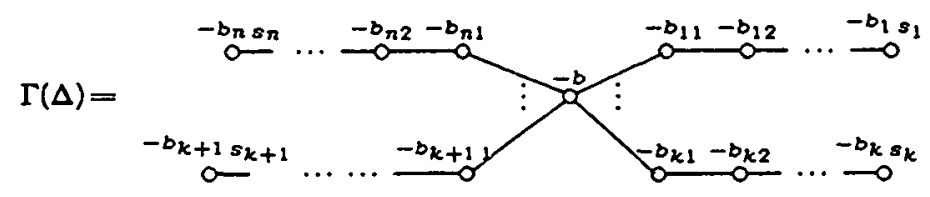

with weights determined as follows. Assume first that no $\alpha_{i}$ is 0 . Then there are unique inlegers $\beta_{i}$ for $i=1, \ldots, n$ and $b \in Z$ satisfying

$$
\sum_{i=1}^{n} \beta_{i} \alpha_{1} \cdots \hat{\alpha}_{i} \cdots \alpha_{n}=-\varepsilon+b \alpha_{1} \cdots \alpha_{n}, \quad 0 \leqslant \beta_{i}<\alpha_{i}
$$

This determines $b$, and the $b_{i j}$ are deternined by

$$
\left[b_{i 1}, \ldots, b_{i s_{i}}\right]=\frac{\alpha_{i}}{\beta_{i}} \text { with } b_{i j} \geqslant 2 \text { for all } i, j
$$


Nole that these equations come directly from Proposition 2.1. However, in practice it is easier to compule the ingredients of (2) as fullows. It is not hard to verify that

$$
\varepsilon \frac{\alpha_{1} \cdots \hat{\alpha}_{1} \cdots \alpha_{n}}{\alpha_{1}}=\left[b_{i}, b_{i s_{i}}, \ldots, b_{i 1}\right]
$$

with

$$
b_{i}=\left\lceil\varepsilon \frac{\alpha_{1} \cdots \hat{\alpha}_{i} \cdots \alpha_{n}}{\alpha_{i}}\right\rceil
$$

The $b_{i j}$ are easily determined from (5) and (6), the $\beta_{i}$ then from (4) or from $\beta_{i} \alpha_{1} \cdots \hat{\alpha}_{i} \cdots \alpha_{n} \equiv-\varepsilon\left(\bmod \alpha_{i}\right)$, and then finally $b$ is the nearest integer to $\sum \beta_{i} / \alpha_{i}$.

If some $\alpha_{i}$ in (1) is 0 then the other $\alpha_{j}$ all equal 1 . We put $\Gamma(\Delta)=\stackrel{0}{0} \stackrel{0}{0}$, which is as in (2) with $b=0$ and with empty chains for the 1-weighted edges of $\Delta$. (Even when no $\alpha_{i}$ is zero the chain in $\Gamma(\Delta)$ corresponding to a 1-weighted edge of $\Delta$ is empty.)

Now suppose

$$
\Delta=\Delta_{1}^{\prime}-\Delta_{2}^{\prime}
$$

is a splice diagram such that

$$
\Delta_{1}=\Delta_{1}^{\prime} \longrightarrow \text { and } \Delta_{2}=\sigma \Delta_{2}^{\prime}
$$

are splice diagrams for which we have already constructed weighted trees

$$
\Gamma\left(\Delta_{1}\right)=\Gamma_{1}^{\prime}-\cdots-b_{1}^{-b_{1}} \text { and } \Gamma\left(\Delta_{2}\right)=-c_{1} \cdots-\Gamma_{2}^{\prime}
$$

Consider $\Gamma\left(\Delta_{1}\right)$ and $\Gamma\left(\Delta_{2}\right)$ as rooted trees, wilh the $\left(-b_{1}\right)$ - and $\left(-c_{1}\right)$-weighted verlices as roots. We have $\operatorname{cf} \Gamma^{\prime}\left(\Delta_{1}\right)=1 /\left(-b_{0}\right)$ and $\mathrm{cf} \Gamma\left(\Delta_{2}\right)=1 /\left(-c_{0}\right)$ for some $b_{0}, c_{0} \in Z$. We put

$$
\Gamma(\Delta)=\Gamma_{1}^{\prime} \cdots-b_{1}^{-b_{0}-c_{0}-c_{1}} \cdots-\Gamma_{2}^{\prime} .
$$

In this way we can construct $\Gamma(\Delta)$ inductively for any normal form splice diagram. We call this procedure of pulting $\Gamma(\Delta)$ logether out of simpler trees splicing.

Remarks. (i) It is not hard to check that the above numbers $b_{0}$ and $c_{0}$ are the same as the numbers appearing in (6), so they do not need to be re-calculated.

(ii) The pictures in (9) and (10) are slightly misleading if the indicated edge of $\Delta_{1}$ has edge weight 1 , since then the appropriate chain of $\Gamma\left(\Delta_{1}\right)$ is empty and the 
$\left(-b_{1}\right)$-weighted vertex is the "central vertex" in (2), usually of valency $>1$. The same remark holds for $\Delta_{2}$.

We must show that our construction gives a unimodular weighted tree $\Gamma$ with $\Delta(\Gamma)=\Delta$. This involves computation of determinants of subtrees, for which we may use continued fractions of sublrees, as in Section 2. It thus suffices to observe that, when computing the continued fraction of a subtree which contains the right half of $\Gamma(\Delta)$ with respect to a root vertex to the left of the $\left(-b_{0}\right)$-weighted vertex, the part to the right of the $\left(-b_{1}\right)$-weighted vertex contributes nothing, since

$$
\operatorname{cr}\left(\stackrel{-b_{0}-c_{0}-c_{1}}{-} \cdots-\left[-\left[\Gamma_{2}^{\prime}\right]=\left[-b_{0},-c_{0},-\frac{1}{c_{0}}\right]=\infty,\right.\right.
$$

and sinularly with right and left exchanged. This olsservation also shows that $\Gamma(\Delta)$ does not depend on the order in which we "splice together" its pieces.

We have seen that $\Delta(\Gamma(\Delta))$ is equal to $\Delta$ if $\Delta$ is in normal form. The combinatorial proof of Theorem 3.1 is complete if we show that a unimodular weighted tree $\Gamma$ in normal form is determined by its splice diagram $\Delta=\Delta(\Gamma)$. Given $\Delta$, Proposition 2.1 determines the continued fractions of the extremal chains of $\Gamma$ and hence determines these chains. With slightly more effort a similar argument shows that once all but one chain at a given node of $\mathrm{I}$ have been determined, the weight at that node and the last chain at that node are also determined. Thus, inductively, $\mathrm{\Gamma}$ is determined.

Note that the weighted tree $\Gamma(\Delta)$ that we construct above is usually not in normal form, since the weights $-b_{0}$ and $-c_{0}$ introduced in the splicing step need not be $\leqslant-2$. In parlicular, $\Gamma(\Delta)$ will usually not have minimal $p_{+}$in its equivalence class. Ilowever, it is easy to determine $p_{+} \Gamma(\Delta)$, and in the next section we shall use this to deduce the minimal $p_{+}$for the equivalence class.

LEMMA 3.2. Let $\Delta$ be a connecled normal form splice diagram and let $\nu$ be the number of nodes of $\Delta$ which either have vertex weight -1 or which have an adjacent edge weight 0 . Let $\sigma$ be the number of edges of $\Delta$ connecting two nodes. Then

$$
p_{+} l^{\prime}(\Delta)=\nu+\sigma
$$

Proof: We apply Corollary 2.4. Suppose first that $\Delta$ is as in (1) so $\Gamma=\Gamma(\Delta)$ is as in (2). If some $\alpha_{i}$ is 0 then $\Gamma=0 \quad 00$ which has $p_{+}=1$ as claimed. Otherwise we take the central vertex $v$ of $\Gamma$ as root vertex and then the $c f \Gamma_{u}$ appearing in Corollary 2.4 are all negative except maybe $c \Gamma^{\prime}{ }_{v}=\mathrm{cf} \Gamma^{\prime}=-\varepsilon /\left(\alpha_{1} \cdots \alpha_{n}\right)$. 'Thus the Lenma holds in this case. On the other hand, if $\Gamma$ is obtained by splicing $\Gamma_{1}$ and $\Gamma_{2}$ as in the proof of Theorem 3.1, then, as observed there, the relevant set of continued fractions $\mathrm{c} \Gamma \Gamma_{u}$ for $\Gamma$ consists of the sets for $\Gamma_{1}$ and $\Gamma_{2}$ and an additional 0 and $\infty$, so $p_{+} \Gamma=p_{+} \Gamma_{1}+p_{+} \Gamma_{2}+1$. The lemma follows. 
We shall need the following lemma later. It follows directly from the construction of $\Delta(\Gamma)$.

LEMmA 3.3. For any unimodular forest $\Gamma$, the normal form splice diagrams for $\Gamma$ and $-\Gamma$ differ only in the signs of the vertex weights of the vertices which have no adjacent zero edge weight.

\section{Minimal $p_{+}$AND DEFINITE UNIMOdUlaR TREeS}

Let $\Delta$ be a connected normal form splice diagram. Let $\Delta_{0}$ be the full subtree on the set of $(-1)$-weighted nodes and $\delta_{0}$ the number of components of $\Delta_{0}$. Let $\delta_{1}$ be the number of edges with negative edge determinant connecting two (+1)-weighted nodes.

THEOREM 4.1. The normal form unimodular tree $\Gamma$ with splice diagram $\Delta$ has $p_{+} \Gamma=\delta_{0}+\delta_{1}$, so any weighted tree in the equivalence class has $p_{+}$at least this large.

Proof: The normal form tree $\Gamma$ is obtained by reducing the tree $\Gamma(\Delta)$ described in the previous section. Lemma 3.2 tells us $p_{+}$before reduction, so we must compute how much this reduction decreases $p_{+}$. We shall call our weighted tree $\Gamma$ at every stage of the process.

Let

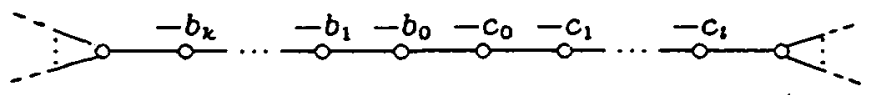

be a maximal chain connecting two nodes of $\Gamma$, with notation as in the previous section. The only way this part of $\Gamma$ may not be in normal form is that one or both of $-b_{0}$ and $-c_{0}$ may exceed -2 . Let the corresponding edge of $\Delta$ be

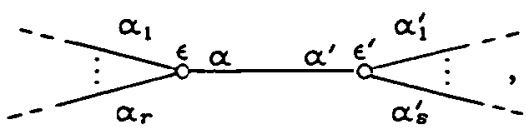

and write $\beta=\alpha_{1} \cdots \alpha_{r}, \beta^{\prime}=\alpha_{1}^{\prime} \cdots \alpha_{s}^{\prime}$, so the edge determinant is det $=\varepsilon \varepsilon^{\prime} \alpha \alpha^{\prime}-\beta \beta^{\prime}$. Note that the description of $b_{0}$ and $c_{0}$ in the previous section implies that sign $b_{0}=\operatorname{sign} \varepsilon$ unless $\alpha \beta=0$, in which case $b_{0}=0$, and similarly for $c_{0}$. We must distinguish several cases. In cases 1 to 4 we assume that none of $\alpha, \alpha^{\prime}, \beta, \beta^{\prime}$ is zero, so $b_{0}$ and $c_{0}$ are non-zero.

Case 1. $\varepsilon=\varepsilon^{\prime}=+1$, det $>0$. Since $\left[b_{0}, \ldots, b_{k}\right]=\beta / \alpha$ and $\left[c_{0}, \ldots, c_{\ell}\right]=\beta^{\prime} / \alpha^{\prime}$, the inequality det $>0$ is equivalent to

$$
\left[b_{0}, \ldots, b_{k}\right]\left[c_{0}, \ldots, c_{\ell}\right]<1
$$


This implies that at least one of $b_{0}$ and $c_{0}$ equals 1 , say $b_{0}=1$. Then we can perform a (-1)-blowdown to get

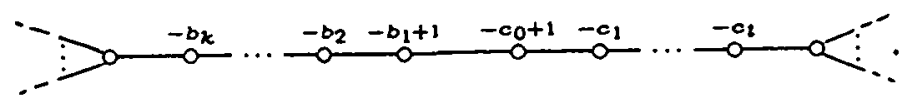

Now $\left[b_{1}-1, b_{2}, \ldots, b_{k}\right]=\beta /(\alpha-\beta)$ and $\left[c_{0}-1, c_{1}, \ldots, c_{1}\right]=\left(\beta^{\prime}-\alpha^{\prime}\right) / \alpha^{\prime}$, and the inequality $\beta / \alpha \cdot \beta^{\prime} / \alpha^{\prime}<1$ is equivalent to $\beta /(\alpha-\beta) \cdot\left(\beta^{\prime}-\alpha\right) / \alpha^{\prime}<1$. 'Thus the analogue of inequality (3) holds for this new chain (4) and either $c_{0}-1=0$ or we can repeat the argument. After finitely many repetitions we must create a weight 0 , and a 0 -absorption then completes the reduction. Since we have performed several (-1)-blowdowns followed by a 0 -absorption, the effect on $p_{+}$is to decrease it by 1 .

Case 2. $\varepsilon=\varepsilon^{\prime}=+1$, det $<0$. In this case inequality (3) is reversed and implies that at least one of $b_{0}$ and $c_{0}$ exceeds 1 . A (-1)-blowdown may be possible, but it cannot create a 0 weight; moreover, the analogue of the reverse inequality to (3) then still holds for the result. Inductively, the same is true for any further (-1)-blowdowns that become possible. 'Thus reduction is completed after finitely many (-1)-blowdowns, and $p_{+}$is not affected.

'l'he remaining cases are easier since the reduction can be described explicitly in each one.

Cnse 3. $\varepsilon$ and $\varepsilon^{\prime}$ have opposite sigus, say $-\varepsilon=\varepsilon^{\prime}=+1$. Then $\operatorname{det}<0$. In this case $b_{0}$ is negative and we can perform the reduction by doing $\left|b_{0}\right|(-1)$-blowups next to the $\left(-b_{0}\right)$-weighted vertex followed by a 0 -absorption to reach

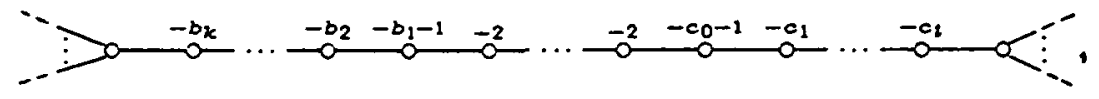

with $\left|b_{0}\right|-1$ vertices weighted $(-2)$ in the nidule. The effect on $p_{+}$is to reduce it by 1.

Case 4. $\varepsilon=\varepsilon^{\prime}=-1$. We proceed as in Case 3 to obtain (5). At this point $c_{0}+1$ is non-positive so we do $\left|c_{0}\right|-1(-1)$-blowups next to it followed by a 0 -absorption to complete the reduction. The effect on $p_{+}$is to reduce it by 2 .

The final cases are those in which al least one of $\alpha, \alpha^{\prime}, \beta, \beta^{\prime}$ is zero. With no loss of generality it is one of $\alpha$ and $\beta$. Note that $\alpha=0$ cannot occur with $\beta^{\prime}=0$ since det $\neq 0$.

Case 5. $\alpha=0, \varepsilon^{\prime}=+1$. Then det $<0$. Also $\varepsilon=+1$ by definition of normal form. In this case the chain (1) is 


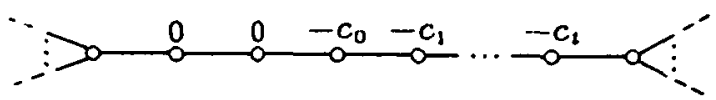

and a 0 -absorption possibly followed by some $(-1)$-blowdowns does the reduction; $p_{+}$ is reduced by 1 .

Case 6. $\alpha=0, \epsilon^{\prime}=-1$. The relevant chain is (6) with $-c_{0}$ positive, so we need a 0 -absorption followed by the procedure of Case 3 , reducing $p_{+}$by 2 ,

Case 7. $\alpha=0, \alpha^{\prime}=0$. Two 0 -absorplions suflice, reducing $p_{+}$by 2 .

Case 8. $\beta=0$. In this case the chain (1) is

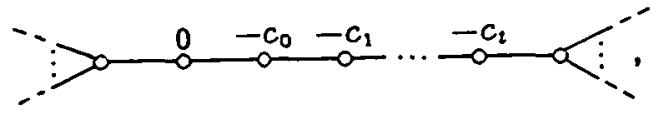

so a single 0 -absorption suffices, reducing $\boldsymbol{p}_{+}$by 1 .

Now, for $i=1, \ldots, 8$, let $C_{i}$ denote the number of edges of $\Delta$ that are in Case $i$. Then by Lemma 3.2 and the above, the normal form $\Gamma$ has $p_{+}$equal to

$$
p_{+}=\nu+\rho-C_{1}-C_{3}-2 C_{4}-C_{5}-2 C_{6}-2 C_{7}-C_{8} .
$$

But $C_{5}+C_{6}+2 C_{7}$ is the number of nodes with an adjacent 0 -weight, so $\nu-$ $\left(C_{5}+C_{6}+2 C_{7}\right)$ is the number of $(-1)$-weighted nodes. Since $C_{4}$ is the number of edges connecting two $(-1)$-weighted nodes, $\nu-\left(C_{5}+C_{6}+2 C_{7}\right)-C_{4}$ is the number of components of the full subgraph on the $(-1)$-weighted nodes, that is

$$
\delta_{0}=\nu-C_{4}-C_{5}-C_{6}-2 C_{7} .
$$

On the other hand, the number of edges with negative edge determinant connecting two $(+1)$-weighted nodes (recall that nodes with an adjacent $U$-weight are $(+1)$-weighled by the definition of normal form) is

$$
\delta_{1}=\rho-C_{1}-C_{3}-C_{4}-C_{6}-C_{8} .
$$

Combining (8), (9), and (10) proves the theorem.

Corollary 4.2. [3, Theorem 9.4]. A normal form unimodular tree is negative definite if and only if its splice diagram has only positive edge weights, vertex weights, and edge determinants. Every negative definite unimodular tree is obtained from a normal form one by repeated (-1)-bluwups.

Proof: By Theorem 4.1, all vertex weights are +1 and all edge determinants are positive. The edge weights are non-negative by definition of norınal form and no edge has a zero edge-weight since it would then have negative edge determinaut. The second sentence follows from Corollary 1.3. 


\section{Semi-DEFINITE UNimodular trees}

As remarked in the introduction, both negalive definite and negative semi-definite unimodular trees are of geometric interest. We described the definite ones above, and we describe the semi-definite ones $\left(p_{+}=1\right)$ here. The discussion is a model for describing the unimodular trees with any prescribed $p_{+}$.

Suppose $\Gamma$ is a negative semi-definite unimodular tree. Let $\Gamma_{v}$ be the corresponding normal form weighted forest (it may not be a tree). Then $p_{+} \Gamma_{0} \leqslant p_{+} \Gamma=1$, so $p_{+} \Gamma_{0}=0$ or 1 .

Case 1. $p_{+} \Gamma_{0}=1$. This is the more interesting case, being the only one that can occur for configuration diagrams of divisors in compact complex surfaces ([6, Theorem $5.3],[7])$. It is also the simpler case $-\Gamma$ is obtained from $\Gamma_{0}$ by repeated $(-1)$-blowups by Corollary 1.3. In particular, $\Gamma_{0}$ is a tree. By Theorem 4.1 there are two basic possibilities for the normal form splice diagram $\Delta$

- all vertex weights equal +-1 and exactly one edge delerminant is negative;

- the full subgraph $\Delta_{0}$ on the (-1)-weighted nodes is connected and nonempty and no edge connecting nodes outside $\Delta_{0}$ has negative edge determinant.

A slightly surprising example is that the second of these possibilities holds when $\Gamma_{0}$ is the normal form for a positive definite unimodular tree $\Gamma_{1}$. Indeed, $\Delta$ is obtained from the normal form splice diagram for the negative definite tree $-\Gamma_{1}$ by changing all vertex weights to -1 (Lemma 3.3 ).

Case 2. $p_{+} \Gamma_{0}=0$. We could give as precise a description of $\Gamma$ as in Case 1 , but it is more complicated and not worth the effort. Instead we just describe $\Gamma$ up to strict equivalence (repeated (-1)-blowups and -blowdowns).

Proposition 5.2. $\Gamma$ is strictly equivalent either to the result of performing a 0 extrusion (reverse of a 0 -absorption) on $\Gamma_{0}$ (so $\Gamma_{0}$ is a tree) or the result of performing a single connecting operation on a forest strictly equivalent to $\Gamma_{0}$.

The second case of this proposition is a bit unsatisfactory-even up to strict equivalence, given $\Gamma_{0}$, there are infinitely many possibilities for $\Gamma$. The reader can easily create many examples like the fullowing one, formed by connecting two non-reduced negalive definite trees. This example has $\Gamma_{0}=0$.

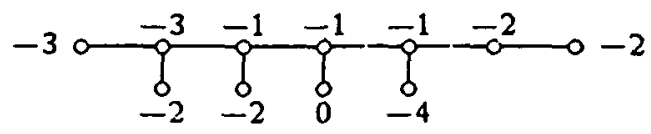

Proof of 5.2: We may assume $\Gamma$ admits no (-1)-blowdowns. Then, since $\Gamma$ is not in normal form, it has a non-negative weight on some cliain. By performing (-1)- 
blowups next to this weight we can make it equal to 0 and 0 -absorption or splitting then turns $\Gamma$ into a tree with minimal $p_{+}$, which can therefore be reduced to $\Gamma_{0}$ by (-1)-blowdowns. Thus $\Gamma$ resulted from $\mathrm{l}_{0}$ by $(-1)$-blowups followed by a connecting operation or 0 -extrusion followed by $(-1)$-blowdowns. If the operation creating the 0 weight was a connecting operation we are done. If it was a 0 -extrusion then the result follows from the following lemma.

Lemma 5.3. Any sequence of (-1)-blowup and-blowdown operations followed by a 0-extrusion on one chain of a weighted graph can be replaced by a 0 -extrusion followed by (-1)-blowups and blowdowns.

Proof: It is enough to show that a single (-1)-blowup or -blowdown operation followed by an adjacent 0 -extrusion can be replaced by a 0 -extrusion followed by $(-1)$-blowups and -blowdowns. Note first that by a (-1)-blowup followed by a (-1)-blowdown we can change

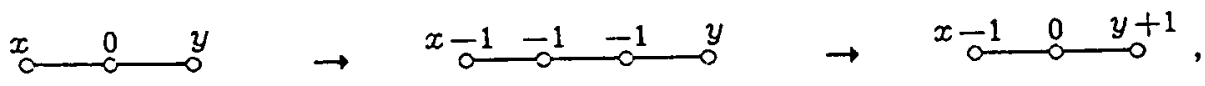

so by a sequence of (-1)-blowups and blowdowns we can do

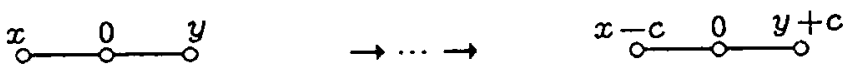

for any $c$. We can replace a $(-1)$-blowup followed by a 0 -extrusion

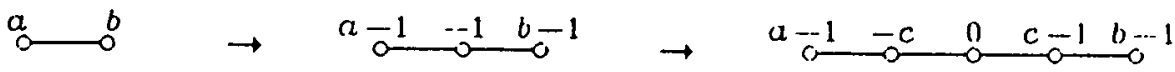

as follows

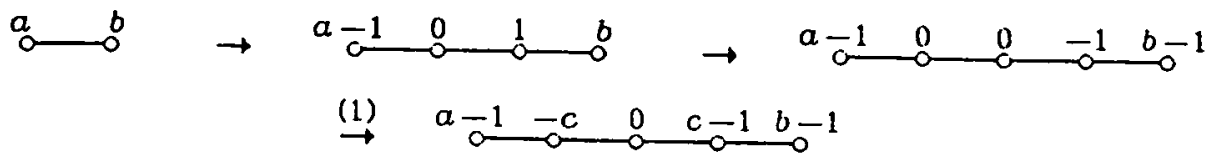

A (-1)-blowdown followed by a 0 -extrusion

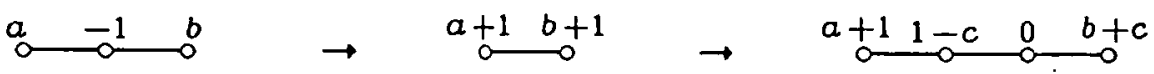

can be replaced by

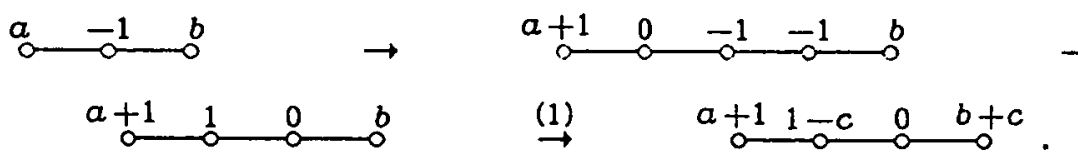

The arguments in the proof of 5.2 and 5.3 can be applied iteratively to prove the following Proposition, which helps describe trees with larger $p_{+}$up to strict equivalence. 
Proposition 5.4. If $\Gamma_{1}$ and $\Gamma_{2}$ are equivalent trees which differ only in one chain and which have the same $p_{+}$then they are strictly equivalent.

\section{Negative DEfinite UNIMOdULAR FORMS REPRESENTED BY TREES}

In this section "form" will always mean unimodular symmetric bilinear form over Z. As pointed out in the Introduction, the classification of indefinite forms is very simple, so only in the definite case is the form represented by a unimodular tree of intrinsic interest. A nice introduction to the classification is in Serre's book [11], from which inuch of the following information is taken.

A negative dcfinite form decomposes uniquely as an orthogonal sum of irreducible ones. The number of isomorphism classes of irreducible ones in dimension $\boldsymbol{n}$ is grows extremely fast with $n$. The same is true for even definite forms (a form $A$ is even if $A(\boldsymbol{x}, \boldsymbol{x})$ is always even), except that they only occur in dimensions $n$ divisible by 8 . For example there are over 80,000,000 even negative definite forms in dimension 32 . However, for small $n$ the classification is tractable, for example, Kneser [4] classified defiuile forms up to $n=16$ (there is one irreducible even form $\Lambda_{n}$ in each dimension $n=8,16$ and one irreducible odd form $\Theta_{n}$ in each dimension $n=1,12,14,15,16$ ), Niemeier [9] classified the even definite forms with $n=24$ (there are 24, of which 2 are reducible), and Conway and Sloane $[1,2]$ have continued Kneser's classification to $n=23$.

It is not hard to represent all the negative definite forms up to rank 16 by trees except for $\Lambda_{8} \oplus \Lambda_{8}$, which is probably not so representable, thought it can of course be represented by a two component forest. 1 do not know how many of Niemeier's 24 forms are representable - the famous Leech lattice, which is one of them, is not.

An ad hoc study of the unimodular forms represented by trees turns up interesting patterns. We restrict to the simplest kind of definite unimodular tree, namely the normal form tree $\Gamma(p, q, r)$ whose splice diagrain is

$$
\Delta(p, q, r)=\propto \frac{p}{q \varphi^{r}} \circ .
$$

This tree has the shape

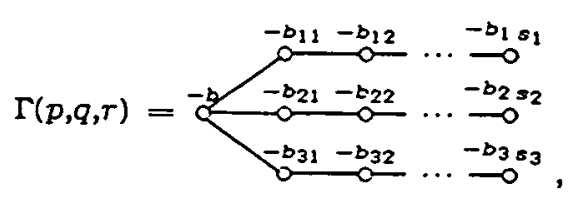

which we also write as $\Gamma\left(b ; b_{11}, \ldots, b_{1 s_{1}} ; b_{21}, \ldots, b_{2 a_{2}} ; b_{31}, \ldots, b_{3 s_{3}}\right)$. We shall denote the corresponding form by $A(p, q, r)$. 
There are many periodic families, for example

$$
\begin{array}{rlr}
A(p, q, r+n p q) & \cong A(p, q, r) \oplus n\langle-1\rangle & (n \geqslant 0), \\
A(2,4 n-1,4 n+1) & \cong \Lambda_{8} \oplus(n-1)(-1) & (n \geqslant 1), \\
A(2 n-1,2 n, 2 n+1) & \cong(2 n+1)\langle-1\rangle & (n \geqslant 2), \\
A(6 n-5,6 n-3,6 n-2) & \cong(3 n+1)(-1\rangle & (n \geqslant 2), \\
A(6 n-2,6 n-1,6 n+1) \cong(3 n+2)\langle-1\rangle & (n \geqslant 1), \\
A(6 n-1,6 n+1,6 n+2) \cong \Lambda_{8} \oplus(3 n-1)\langle-1\rangle & (n \geqslant 1), \\
A(6 n+2,6 n+3,6 n+5) \cong \Lambda_{8} \oplus(3 n)\langle-1\rangle & (n \geqslant 0), \\
A(4 n-1,4 n+1,4 n+3) \cong \Theta_{12} \oplus(n-1)\langle-1\rangle & (n \geqslant 1) \\
A(4 n-3,4 n-1,4 n+1) \cong(n+4)(-1\rangle & (n \geqslant 2),
\end{array}
$$

et cetera, where $n\langle-1\rangle$ is the rank $n$ diagonal form. There are also interesting sequences of examples, such as the following sequences of even forms of rank $8 n$

$A(2,4 n-1,8 n-3) ; A(2,8 n-5,12 n-7) ; A(2 n, 4 n-1,4 n+1) ; A(4 n-1,4 n, 8 n-1)$.

In addition, forms with related properties sometimes seem to be assembled out of similar building blocks. This phenomemon is clearly visible in the following list of trees of type $\Gamma(2, p, q)$ with form isomorphic to $\Lambda_{8} \oplus n\langle-1\rangle$ for some $n$. It includes all exanıles with $p<31$ which are not a consequence of one of the first two periodic families mentioned above, plus an exanple with $p=33$.

$$
\begin{aligned}
& \Gamma(2,11,19)=\Gamma(2 ; 2 ; 6,2 ; 4,2,2,2,2,2) \\
& \Gamma(2,13,21)=\Gamma(2 ; 2 ; 4,2,2,2 ; 6,2,2,2) \\
& \Gamma(2,19,27)=\Gamma(2 ; 2 ; 2,2,7 ; 2,2,2,2,4,2) \\
& \Gamma(2,21,29)=\Gamma(2 ; 2 ; 2,2,2,2,5 ; 2,2,6,2) \\
& \Gamma(2,19,33)=\Gamma(2 ; 2 ; 10,2 ; 3,3,2,2,2,2,2) \\
& \Gamma(2,23,37)=\Gamma(2 ; 2 ; 3,3,2,2,2 ; 10,2,2,2) \\
& \Gamma(2,23,39)=\Gamma(2 ; 2 ; 5,3,2 ; 4,3,2,2,2,2) \\
& \Gamma(2,25,4\rfloor)=\Gamma(2 ; 2 ; 4,3,2,2 ; 5,3,2,2,2) \\
& \Gamma(2,25,43)=\Gamma(2 ; 2 ; 3,5,2 ; 8,2,2,2,2,2) \\
& \Gamma(2,29,47)=\Gamma(2 ; 2 ; 8,2,2,2 ; 3,5,2,2,2) \\
& \Gamma(2,27,35)=\Gamma(2 ; 2 ; 6,2,3 ; 4,2,2,2,2,3) \\
& \Gamma(2,29,37)=\Gamma(2 ; 2 ; 4,2,2,2,3 ; 6,2,2,3) \\
& \Gamma(2,27,47)=\Gamma(2 ; 2 ; 14,2 ; 3,2,3,2,2,2,2,2) \\
& \Gamma(2,33,53)=\Gamma(2 ; 2 ; 14,2,2,2 ; 3,2,3,2,2,2)
\end{aligned}
$$


The phenomenon is visible as far as we have computed also for the $\Gamma(2, p, q)$ representing $\Lambda \oplus n\langle-1\rangle$ for $\Lambda=\Theta_{12}$ and for $\Lambda=\Lambda_{16}$, but not for $\Lambda=\langle-1\rangle$.

Another example of the phenomenon is Yamada and Matsumoto's surprising construction, in terms of certain basic building blocks, of all trees of type $\Gamma(2, p, q)$ which have even forms ([14]).

\section{REFERENCES}

[1] J.H.C. Conway and N.J.A. Sloane, 'On the enumeration of lattices of determinant 1 ', $J$. Number Theory 15 (1982), 83-94.

[2] J.H.C. Conway and N.J.A. Sloane, 'The unimodular lattices of dimension up to 23 and the Minkowski-Siegel mass constanls', European J. Combin. 3 (1982), 219-231.

[3] D. Eisenbud and W.D. Neumann, Three-Dimensional Link Theory and Invariants of Plane Curve Singularities, Annals of Math. Studies 110 (Princeton Univ. Press, 1985).

[4] M. Kneser, 'Klassenzahlen definiter quadralischer Formen', Arch. Math. 8 (1957), 241-250.

[5] W.D. Neumann, 'A calculus for plumbing applied to the topology of complex surface singularities and degenerating complex curves', Trans. Amer. Math. Soc. 208 (1981), 299-343.

[0] W.D. Neumann, 'On the topology of curves in complex surfaces': Proceedings of Conference on Topological Methods in Algebraic Transformation Groups. Progress in Math (Birkhäuser Verlag). (to appear) .

[7] W.D. Neumanm, 'Links of curves in compact complex surfaces', (in preparation).

[8] W.D. Neumann and D. Zagier, 'A note on an invariant of Fintushel and Stern': Proc. Special Year in Topology Maryland 1983/4. Lecture Notes in Mathematics 1167, pp. 241-244 (Springer-Verlag, Berlin, Heidelberg, New York).

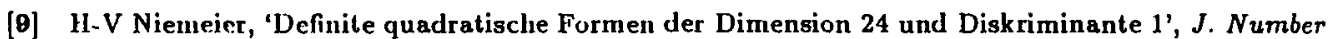
Theory 5 (1973), 142-178.

[10] T. Pelrie and ' $\Gamma$. tom Dieck, 'HIomulogy planes and announcement and survey': Proceedings of Conference on Topological Methods in Algebraic Transformation Groups. Progress in Math (Birkhäuser Verlag). (to appear) .

[11] J.P. Serre, $A$ Course in Arithmetic, Graduate Texts in Mathematics 7 (Springer-Verlag, Berlin, Heidelberg, New York, 1973).

[12] L. Siebenmann, On the vanishing of the Rohlin inveriant and non-finitely amphicheiral homology 3-spheres: Topology Symposium, Siegen 1979, Koschorke and Neumann, ed.. Lecture Notes in Mathematics 788, pp. 172-222 (Springer-Verlag, Berlin, Heidelberg, New York).

[13] F. Waldhausen, 'On irreducible 3-manifulds that are sufficiently large', Ann. of Math. 87 (1968), 56-88.

[14] Akio Yamada and Yukio Matsumoto, ' $O$ n the resulution diagrams of Brieskorn singularities $(2, q, r)$ of type II', Canad. J. Math. 35 (1983), 1049-1058.

Departinent of Mathematics

Ohio State University

Columbus, OH 43210

United States of America 